tests underwent coronary angiography. Patients were followed up from 6-18 months in both groups

Results During the study period there were 172 patients in the CCSE and 162 in the CPSE group. Majority were referred for CPRO (120 vs.108, $\mathrm{p}=0.5)$. Image quality was similar in both groups $(p=0.20)$. The number of positive stress test was CCSE 20 vs. CPSE $18(\mathrm{p}=0.09)$ (Table1). Coronary angiography was performed in 18 patients in both groups, of which 15 had positive tests (Two patients were not investigated further in CCSE group as decision made by consultant for medical management only). Sensitivity and specificity was $73 \%$ and $100 \%$ in the CCSE vs. $83 \%$ and $78 \%$ in CPSE group. Territory correlation was similar in both groups. During the follow up period none of the patients with negative test result in the CCSE group had a coronary angiogram, while 9 patients in CPSE group had a coronary angiogram. Of these only 2 were positive and 7 were negative, thus giving a specificity of $78 \%$ (Table 2).

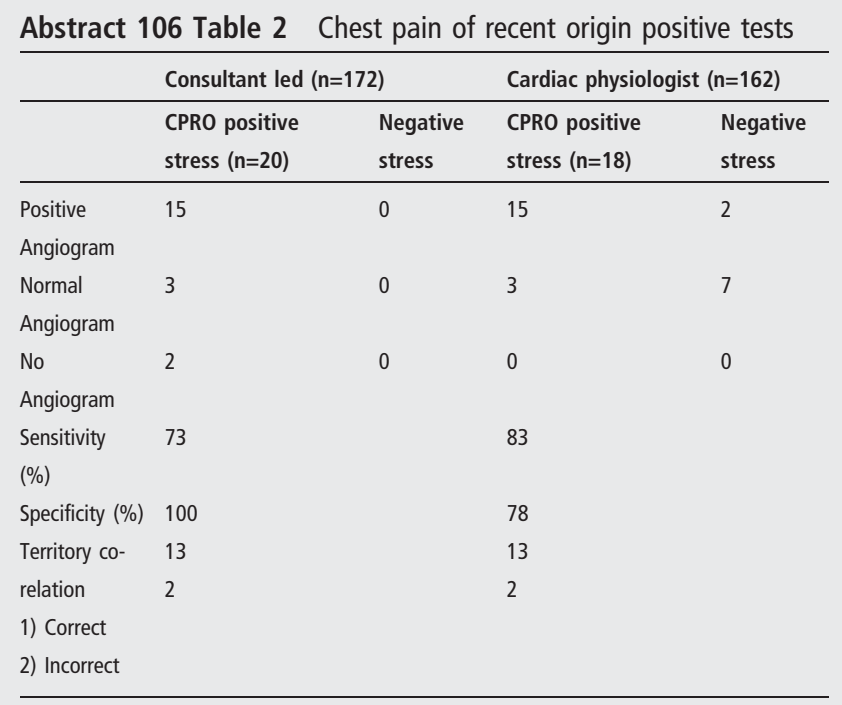

Conclusion A cardiac physiologist managed exercise stress echo service (managing and reporting the test independently) has high sensitivity and specificity. It is a safe and effective way to manage patients referred with CPRO. This will help reduce waiting times and improve efficiency of the service.

Though, it's hindered by a lack of clinical decision making process on the day, which may warrant further investigation by the non-specialist.

\section{THE LONG TERM PROGNOSTIC VALUE OF DIPYRIDAMOLE STRESS MYOCARDIAL CONTRAST ECHOCARDIOGRAPHY IN COMPARISON WITH SINGLE PHOTON EMISSION TOMOGRAPHY IN PATIENTS WITH KNOWN OR SUSPECTED CORONARY ARTERY DISEASE}

${ }^{1}$ Nikos Karogiannis*, 'Jatinder Pabla, 'Sothinathan Gurunathan, 'Anastasia Vamvakidou, ${ }^{1}$ Grace Young, ${ }^{2}$ Roxy Senior. 'Northwick Park Hospital; ${ }^{2}$ Northwick Park Hospital and Royal Brompton Hospital

\subsection{6/heartjnl-2017-311726.106}

Background Single photon emission computed tomography (SPECT) is a well-established method to evaluate patients with coronary artery disease (CAD). Myocardial contrast echocardiography (MCE) is an imaging technique that allows assessment of myocardial perfusion in a real-time setting. Very short term prognostic study has shown that vasodilator MCE is superior to SPECT for the prediction of hard events. We sought to investigate the long term prognostic value of SPECT and MCE in the assessment of patients with known or suspected CAD.

Methods We retrospectively followed-up patients with suspected or known CAD who were scheduled for coronary angiography and who also underwent MCE and SPECT at our

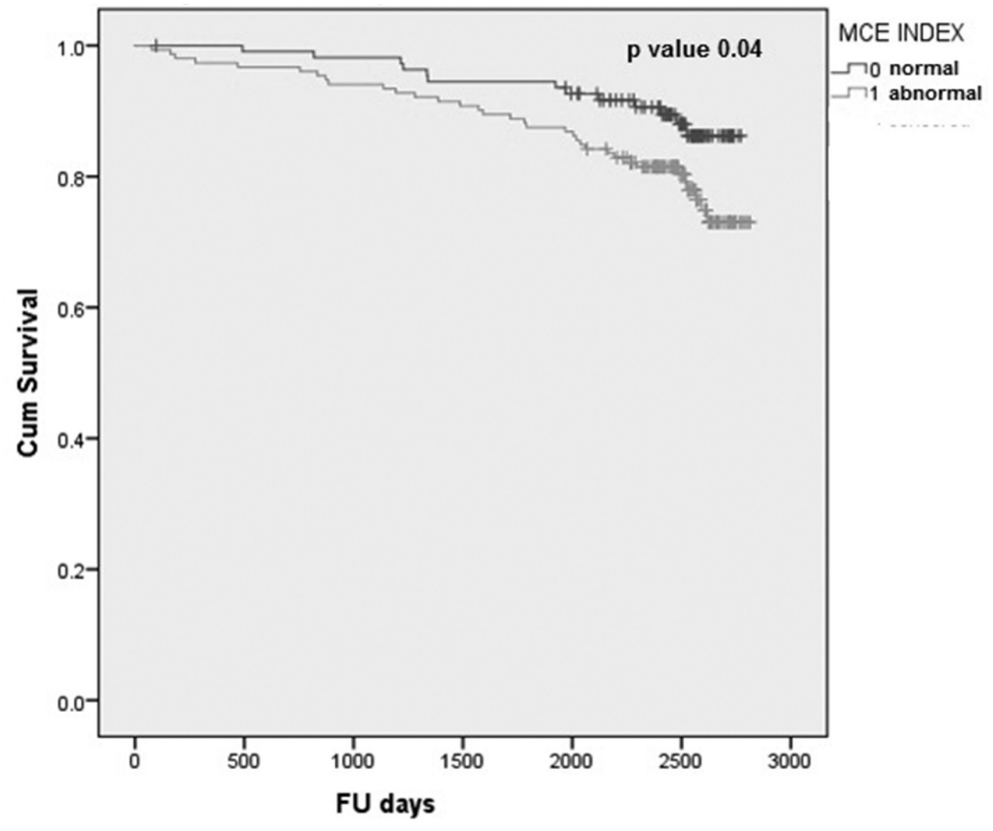

Abstract 107 Figure 1 Kaplan-Meier curve showing the long term prognostic value in predicting all cause-morality and NFMl for normal versus abnormal MCE 
institute, as part of multicentre studies performed between January 2002 and December 2009. Rest and vasodilator SPECT was performed after injection of $99 \mathrm{mTc}$-sestamibi using the standard technique on separate days. Coronary Angiography (CA) was performed within thirty days of stress imaging. We calculated the ratio of the number of abnormal segments (at rest and/or stress) to the total number of segments expressed as $\%$ for both MCE and SPECT in order to obtain a uniform assessment of the total ischaemic and scar burden (MCE and SPECT indexes). This population was followed up in 2016 to obtain a long term prognostic value of MCE and SPECT for hard events, all-cause mortality and non-fatal myocardial infarction (NFMI).

Results Of the 277 patients who were analysed, 262 followed up and 15 were lost to follow up (5.4\%). The mean age was 63.4 years and $186(71.8 \%)$ patients were male, $82(31.7 \%)$ had diabetes, 180 (69.5\%) hypertension, 189 (73\%) dyslipidaemia, $26(10 \%)$ family history of CAD (FHCAD) and 64 (24.7\%) were smokers. Prior CAD (angina, known acute myocardial infarction (AMI) or coronary revascularisation) was present in $178(68.7 \%)$ patients, left ventricular systolic dysfunction in $32(12.4 \%)$ and chronic kidney disease in 16 (6.2\%).

Over a mean follow-up period of 80 months(6.6 years) \pm 6 months, 18 patients suffered NFMI and 29 died $(18 \%$ hard events, annualised hard events 2.7\%).

Both MCE and SPECT indexes were significant predictors on univariate analysis for all-cause mortality $(p=0.008$ and $\mathrm{p}=0.035$ respectively), but MCE index was the only independent predictor for hard events (HR 3.711, 95\% CI(1.1312.14), $\mathrm{p}=0.03)$. Figure 1 demonstrates the Kaplan-Meier curve for the long-term prognostic value in all-cause mortality and NFMI of abnormal versus normal MCE. The annualised event rate for the abnormal MCE is $3.8 \%$ versus $1.0 \%$ for the normal MCE.

Conclusion This is the first study to our knowledge that investigated the long-term prognostic value of SPECT and MCE in patients with suspected or known CAD. MCE was the only independent predictor of hard events. These results further support the routine use of MCE and not SPECT for the long-term prognostication of patients with known or suspected CAD.

\section{CLINICAL OUTCOME AND COST-EFFECTIVENESS OF PERFORMING CARDIAC INVESTIGATIONS IN A VERY LOW LIKEHOOD OF CORONARY ARTERY DISEASE POPULATION ACCORDING TO NICE AND ESC RISK PREDICTION MODELS}

Nikos Karogiannis*, Konstantinos Zacharias, Anastasia Vamvakidou, Sothinathan Gurunathan, Roxy Senior. Northwick Park Hospital

10.1136/heartjnl-2017-311726.107

Background The NICE (National Institute for Health and Care Excellence) guidance for the management of recent-onset chest pain in 2010, recommended no routine cardiac investigations for patients with risk of coronary artery disease (CAD) less than 10\%. The ESC (European Society of Cardiology) guidelines for stable angina in 2013, proposed no further testing in patients with a pretest probability below $15 \%$. The management of patients with very low risk for CAD can be challenging, particularly when there is high clinical suspicion, family history of premature CAD (FHCAD) or ethnicity background with high prevalence of CAD. We sought to evaluate the clinical and economic impact of performing tests to diagnose CAD in this population.

Methods We retrospectively analysed patients with very low risk for $\mathrm{CAD}$ who attended rapid access chest pain clinic (RACPC) due to recent onset chest pain. The likelihood of CAD was estimated by the NICE modified Diamond-Forrester formula and the ESC pretest probability formula and it was below $10 \%$ and $15 \%$ respectively. According to the guidelines, no further investigations are recommended for this group. Patients underwent exercise ECG (ExECG) or/and stress echocardiography (SE) mainly due to increased clinical suspicion. Coronary Angiography (CA) was subsequently performed, if it was clinically indicated. This population was followed-up for all-cause mortality, myocardial infarction (MI) and revascularisation. Cost-analysis was also performed.

Results Over a period of 12 months, 279 patients underwent ExECG $[171(61,3 \%)$ negative, $13(4,6 \%)$ positive and 56 $(20 \%)$ inconclusive] or/and SE [72 (25,8\%) negative and 5 $(1.7 \%)$ positive]. Coronary angiography performed in 26 patients $(9.3 \%)$ and significant CAD was identified in one patient (0.3\%). Regarding risk factors, 88 (31.5\%) patients had FHCAD, 66 (23.6\%) hypertension, 30 (10.7\%) dyslipidaemia, $17(6.1 \%)$ diabetes and $10(3.5 \%)$ were smokers. The ethnicity distribution predominantly consisted of South Asians (49.8\%).

Over 55 months (4.5 years) \pm 5 months of follow up, two patients were diagnosed with myocardial infarction $(0.7 \%)$ but only one had significant $\mathrm{CAD}$ and subsequent revascularisation $(0.3 \%)$. Both patients had ExECG initially which was negative. Also, two patients died $(0.7 \%)$ both from non-cardiovascular cause.

A total cost of $£ 69,060$ was spent in these tests with a mean cost of $£ 247$ per patient.

Conclusion The above findings support the rationale of avoiding further cardiac investigations in patients who are at very low risk of CAD as it is recommended by NICE and ESC guidelines. The cost-effectiveness of this strategy is welldepicted in previous studies and our findings are consistent with these results. Current guidance for the management of these patients seems that it is reassuringly applicable in ethnicities with high prevalence of CAD.

\section{THE PROGNOSTIC VALUE OF STRESS CMR IN A TERTIARY CENTRE}

Mohammed Meah* ${ }^{*}$ Wern Ding, Jonathan Hasleton, James McShane, Joseph Mills. NHS

\subsection{6/heartjnl-2017-311726.108}

Background Stress cardiac magnetic resonance imaging (CMR) is increasingly being used in the assessment of myocardial perfusion at rest and in response to exertion. It provides information on the presence or absence of functionally significant coronary artery disease and allows us to predict the benefit of revascularisation. The aim of this study is to assess the accuracy and prognostic value of stress CMR in our tertiary centre.

Method Retrospective single centre study of every adenosine stress CMR done for patients suspected of having ischaemic heart disease between January 2012 and December 2014 $(n=525)$. Reports were reviewed and patients categorised into 